\title{
AFRL-RX-WP-TP-2012-0397
}

\section{INVERSE PROBLEM FOR ELECTROMAGNETIC PROPAGATION IN A DIELECTRIC MEDIUM USING MARKOV CHAIN MONTE CARLO METHOD (PREPRINT)}

Jeremy S. Knopp

Materials State Awareness \& Supportability Branch

Structural Materials Division

Fumio Kojima

Kobe University

AUGUST 2012

Interim

Approved for public release; distribution unlimited.

See additional restrictions described on inside pages

STINFO COPY

AIR FORCE RESEARCH LABORATORY

MATERIALS AND MANUFACTURING DIRECTORATE

WRIGHT-PATTERSON AIR FORCE BASE, OH 45433-7750

AIR FORCE MATERIEL COMMAND

UNITED STATES AIR FORCE 


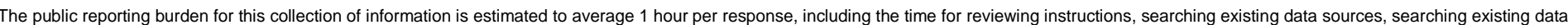

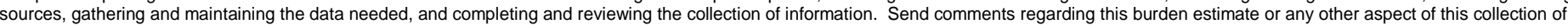

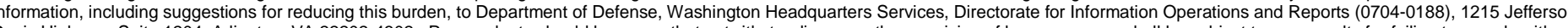

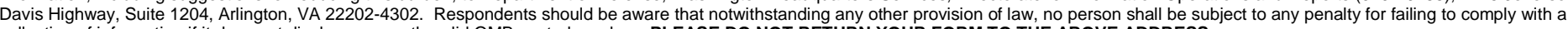
collection of information if it does not display a currently valid OMB control number. PLEASE DO NOT RETURN YOUR FORM TO THE ABOVE ADDRESS.

\begin{tabular}{|l|l|l|}
\hline $\begin{array}{l}\text { 1. REPORT DATE (DD-MM-YY) } \\
\text { August } 2012\end{array}$ & $\begin{array}{c}\text { 2. REPORT TYPE } \\
\text { Technical Paper }\end{array}$ & $\begin{array}{c}\text { 3. DATES COVERED (From - To) } \\
1 \text { July } 2012-1 \text { August } 2012\end{array}$ \\
\hline
\end{tabular}

\section{TITLE AND SUBTITLE}

INVERSE PROBLEM FOR ELECTROMAGNETIC PROPAGATION IN A DIELECTRIC MEDIUM USING MARKOV CHAIN MONTE CARLO METHOD (PREPRINT)

\section{AUTHOR(S)}

Jeremy S. Knopp (AFRL/RXCA)

Fumio Kojima (Kobe University)

\section{5a. CONTRACT NUMBER}

In-house

5b. GRANT NUMBER

5c. PROGRAM ELEMENT NUMBER 62102F

5d. PROJECT NUMBER 4349

5e. TASK NUMBER

40

5f. WORK UNIT NUMBER

X08J

\section{PERFORMING ORGANIZATION NAME(S) AND ADDRESS(ES)}

Materials State Awareness \& Supportability Branch (AFRL/RXCA)

Structural Materials Division

Air Force Research Laboratory, Materials and Manufacturing Directorate

Wright-Patterson Air Force Base, OH 45433-7750

Air Force Materiel Command, United States Air Force

9. SPONSORING/MONITORING AGENCY NAME(S) AND ADDRESS(ES)

Air Force Research Laboratory

Materials and Manufacturing Directorate

Wright-Patterson Air Force Base, OH 45433-7750

Air Force Materiel Command

United States Air Force
8. PERFORMING ORGANIZATION REPORT NUMBER

AFRL-RX-WP-TP-2012-0397

12. DISTRIBUTIONIAVAILABILITY STATEMENT

Approved for public release; distribution unlimited. Preprint to be submitted to International Journal of Innovative Computing Inform...

\section{SUPPLEMENTARY NOTES}

The U.S. Government is joint author of this work and has the right to use, modify, reproduce, release, perform, display, or disclose the work. PA Case Number and clearance date: 88ABW-2011-2803, 19 May 2011. This document contains color.

14. ABSTRACT

This paper is concerned with a stochastic inverse methodology arising in electromagnetic imaging. Nondestructive testing using guided microwaves covers a wide range of industrial applications including early detection of anomalies in conducting materials. The focus of this paper is the identification of electromagnetic material parameters and emphasis is on one dimensional scattering of a dielectric slab. The direct problem can be solved numerically using the finitedifference time-domain method (FDTD). The Markov Chain Monte Carlo method (MCMC) is applied to the inversion problem. Some successful results of computational experiments are demonstrated in order to show the feasibility and applicability of the proposed method.

15. SUBJECT TERMS

Microwave, Dielectric loss, Electrical cable, FDTD, Metropolis-Hasting algorithm

16. SECURITY CLASSIFICATION OF:

a. REPORT

b. ABSTRACT c. THIS PAGE

Unclassified

17.

OF

ABSTRACT:

SAR
NUMBER OF PAGES

10 19a. NAME OF RESPONSIBLE PERSON (Monitor)

Mark Blodgett

19b. TELEPHONE NUMBER (Include Area Code) N/A 


\title{
INVERSE PROBLEM FOR ELECTROMAGNETIC PROPAGATION IN A DIELECTRIC MEDIUM USING MARKOV CHAIN MONTE CARLO METHOD
}

\author{
Fumio KoJima ${ }^{1}$ And JeRemy S. KnopP ${ }^{2}$ \\ ${ }^{1}$ Organization of Advanced Science and Technology \\ Kobe University \\ 1-1, Rokkodai-cho, Nada-ku, Kobe 657-8501, Japan \\ kojima@koala.kobe-u.ac.jp \\ ${ }^{2}$ AFRL/RXLP, Nondestructive Evaluation Branch \\ Air Force Research Laboratory \\ Wright-Patterson AFB, OH 45433, USA
}

Received March 2011; revised July 2011

\begin{abstract}
This paper is concerned with a stochastic inverse methodology arising in electromagnetic imaging. Nondestructive testing using guided microwaves covers a wide range of industrial applications including early detection of anomalies in conducting materials. The focus of this paper is the identification of electromagnetic material parameters and emphasis is on one dimensional scattering of a dielectric slab. The direct problem can be solved numerically using the finite-difference time-domain method (FDTD). The Markov Chain Monte Carlo method (MCMC) is applied to the inversion problem. Some successful results of computational experiments are demonstrated in order to show the feasibility and applicability of the proposed method.

Keywords: Microwave, Dielectric loss, Electrical cable, FDTD, Metropolis-Hasting algorithm
\end{abstract}

1. Introduction. Recently, interest has been growing in structural health monitoring (SHM) related to aging management of large scale systems, such as airplanes, bridges and nuclear power plants. Various kinds of nondestructive testing (NDT) techniques such as ultrasonic, eddy current, thermal are applied to the detection and the characterization of material damage. Combining NDT with simulation is a key component of future structural monitoring technologies. Mathematical descriptions of non-destructive testing (NDT) can be formulated as either a forward or an inverse problem for the physical domain of the inspection. A forward problem represents a real NDT system mathematically using the input and the output relationship with the appropriate admissible class of material parameters, while an inverse problem involves the construction of a method for recovering and/or visualizing material flaw information using the mathematical formulation of the forward problem. Figure 1 demonstrates the overall configuration of the proposed system. In this paper, a stochastic inverse methodology for NDT arising in electromagnetic imaging is investigated. Nondestructive testing using guided microwaves is used in a wide range of industrial applications including early detection of anomalies in conducting materials, analysis of human muscle tissue, etc. The characterization of material corrosion damage continues to be a very challenging problem. The problem is further complicated by the dispersive nature of the insulation covering the corrosion. Estimating the material properties in conventional measurements has been studied extensively [1]. For instance, L. F. Chen et al. have addressed the practical guidance on the development 


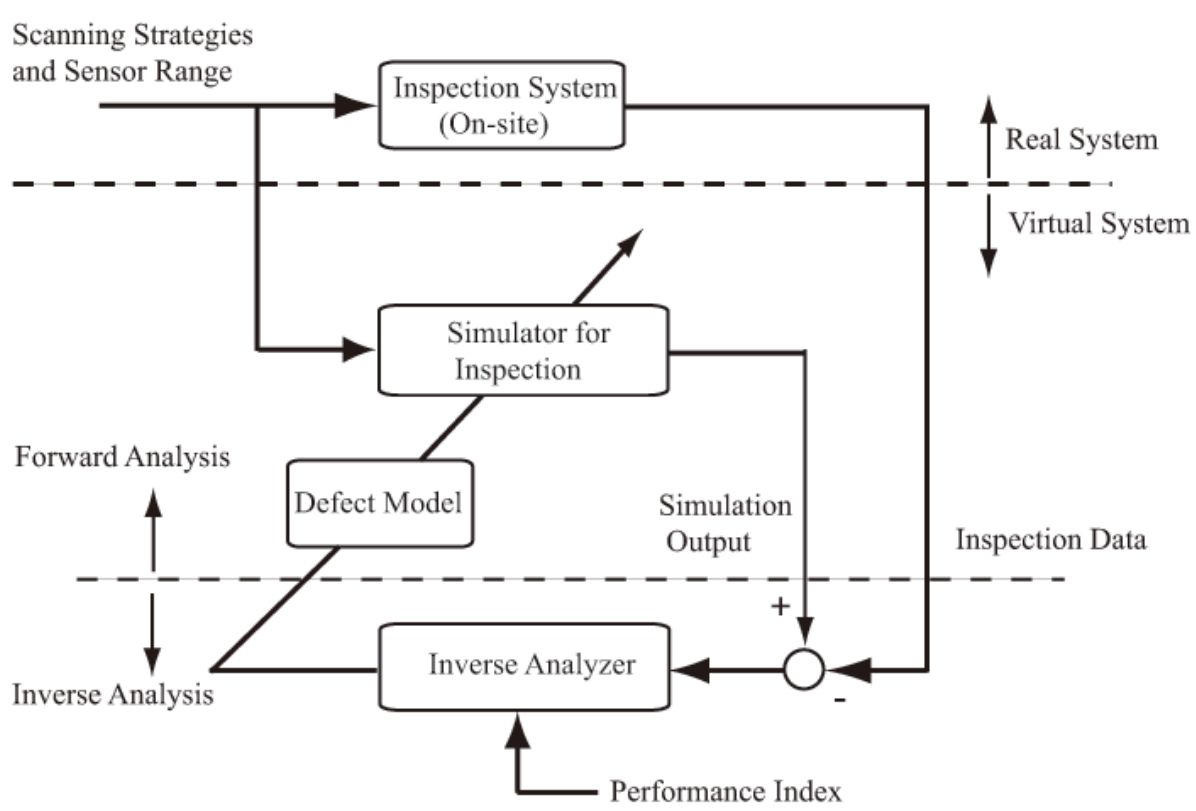

FIGURE 1. Diagnosis system using forward and inverse analysis

of suitable measurement methodologies using micro guided wave for a variety of materials in their book [1]. Our focus in this paper is in the identification of electromagnetic material parameters and the emphasis is on one dimensional scattering of a dielectric slab. Although prior work exists using nonlinear least square methods [2, 3, 4, 5, 6], it is well known that the problem mentioned above has many solutions due to the fact that it is ill-posed. Recently, interest has grown in stochastic inversion using Markov Chain Monte Carlo (MCMC) methods [7, 8]. Although the book written by L. F. Chen [1] et al. contains around thousands of references in a variety of applications, there is not any literature using MCMC to characterize the material properties. The author first proposed the parameter estimation method using the Gibbs sampling algorithm in [9]. The method has great advantages for the more practical aspects of inversions such as setting initial guesses and overcoming local minimums of the inverse solution. In this paper, a stochastic inversion technique based on the Metropolis-Hasting algorithm is applied to our problem.

2. Formulation of the Inverse Problem. Let $D_{x}(t, z), H_{y}(t, z)$ and $E_{x}(t, z)$ be the component of the electric flux density, and the magnetic and electric field at time $t \in[0, T]$ and at location $z \in[0, Z]$. Using normalized electric flux densities and electric fields

$$
\tilde{D}_{x}=\frac{1}{\sqrt{\epsilon_{0} \mu_{0}}} D_{x}, \quad \tilde{E}_{x}=\sqrt{\frac{\epsilon_{0}}{\mu_{0}}} E_{x}
$$

and from Maxwell equations, the electromagnetic propagation in $z$-direction is governed by

$$
\begin{aligned}
\frac{\partial \tilde{D}_{x}(t, z)}{\partial t} & =-\frac{1}{\epsilon_{r} \sqrt{\epsilon_{0} \mu_{0}}} \frac{\partial H_{y}(t, z)}{\partial z}+J_{s}(t, z) \quad \text { in }[0, T] \times[0, Z] \\
\tilde{D}_{x}(\omega, z) & =\epsilon_{r}^{*}(\omega) \tilde{E}_{x}(\omega, z) \\
\frac{\partial H_{y}(t, z)}{\partial t} & =-\frac{1}{\sqrt{\epsilon_{0} \mu_{0}}} \frac{\partial \tilde{E}_{x}(t, z)}{\partial z} \text { in }[0, T] \times[0, Z]
\end{aligned}
$$




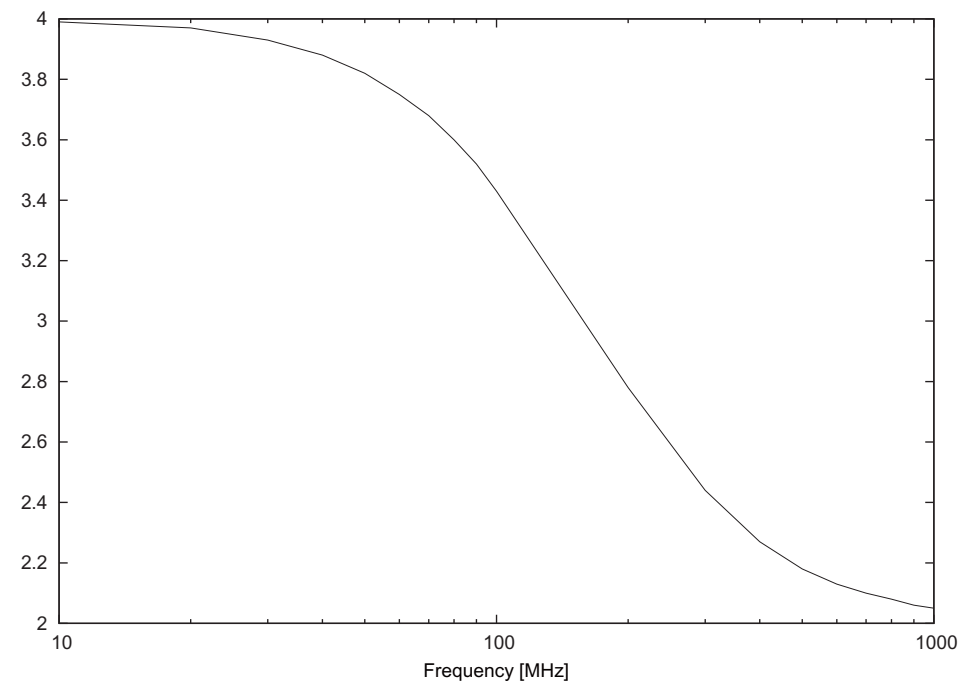

Figure 2. Dielectric constant for a Debye medium over the frequency range of 10 to $1000[\mathrm{MHz}]$

with zero initial states and absorbing boundary conditions. In system (2) and (4), $\epsilon_{0}$ and $\mu_{0}$ denote the dielectric constant and magnetic permeability of vacuum, respectively.

The source current $J_{s}$ is the test signal in the inspection process and is given by

$$
J_{s}(t, z)=\delta\left(z-z_{s}\right) g_{s}(t) I_{\left(0, t_{s}\right)}(t)
$$

where $\delta$ denote the Dirac distribution and $z_{s} \in\left[0, Z_{1}\right)$ denotes the source point of test signal. The test signal is truncated at a finite time $t_{s}$ by the indicator function $I$.

The spatial domain $[0, Z]$ is decomposed into two separate regions. The region $z \in$ $\left[0, Z_{1}\right)$ is air and is assumed to have zero electric polarization and zero conductivity. Hence, in (3), it becomes

$$
\epsilon_{r}^{*}=1 \text {. }
$$

A target material in $z \in\left[Z_{1}, Z\right]$ is assumed to be a chemical material such as a polymer where the dielectric constant and the conductivity are dispersive. A material like this is well approximated by the Debye law described by

$$
\epsilon^{*}(\omega)=\epsilon_{r}+\frac{\sigma}{j \omega \epsilon_{0}}+\frac{\chi_{1}}{1+j \omega \tau}
$$

where $\epsilon_{r}, \sigma, \chi_{1}$ and $\tau$ denote a dielectric constant, conductivity, and frequency-dependent parameters [3]. As indicated in Equation (7), a target material includes a medium whose dielectric constant and conductivity vary over the frequency range. Figures 2 and 3 show a relative dielectric constant and conductivity as functions of frequency for a Debye medium (7) with properties (21). From (7), it follows that the dielectric constant for a Debye medium can be represented as

$$
\epsilon_{r}+\frac{\chi_{1}}{1+(\tau \omega)^{2}}
$$

and the frequency dependent conductivity can be rewritten as

$$
\omega \epsilon_{0}\left\{\frac{\sigma}{\epsilon_{0} \omega}+\frac{\chi_{1} \omega \tau}{1+(\tau \omega)^{2}}\right\}
$$

respectively. 


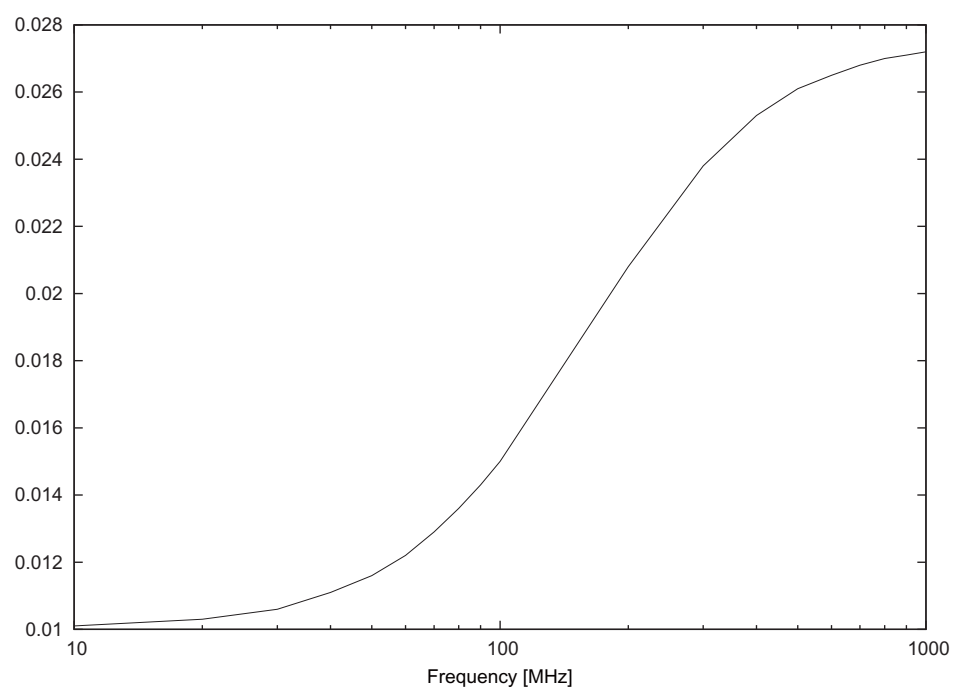

Figure 3. Conductivity for a Debye medium over the frequency range of 10 to $1000[M H z]$

There are many interesting applications for identifying frequency dependent parameters in the Debye model (7). Thus our inverse problem is to identify dielectric parameters

$$
\mathbf{q}=\left\{\epsilon_{r}, \sigma, \chi_{1}, \tau\right\}
$$

from observations near the frequency dependent medium:

$$
Y(t ; \mathbf{q})=E_{x}\left(t, 0 ; J_{s}\right)
$$

where $E_{x}(t, z)$ is the solution of (2)-(4).

3. Numerical Scheme of Direct Problem. The detection signal (8) corresponding to (5) can be obtained by numerical simulation using the finite-difference time-domain (FDTD) method [10]. FDTD uses central-difference approximations to the space and time partial derivatives. The resulting finite-difference equations are solved in a so-called "leapfrog manner". More precisely, the electrical field components in a volume of space are solved at a given instant in time. Then the magnetic filed components in the same spatial volume are solved at the next instant in time.

Taking the central difference formula for both the temporal and spatial derivatives, Equations (2) and (4) can be approximated by

$$
\begin{aligned}
& \frac{\tilde{D}_{x}^{n+1 / 2}[k]-\tilde{D}_{x}^{n-1 / 2}[k]}{\Delta t} \\
= & -\frac{1}{\epsilon_{r} \sqrt{\epsilon_{0} \mu_{0}}} \frac{H_{y}^{n}[k+1 / 2]-H_{y}^{n}[k-1 / 2]}{\Delta z}+J_{s}^{n}[k] \\
& \frac{H_{y}^{n+1}[k+1 / 2]-H_{y}^{n}[k+1 / 2]}{\Delta t} \\
= & -\frac{1}{\sqrt{\epsilon_{0} \mu_{0}}} \frac{\tilde{E}_{x}^{n+1 / 2}[k+1]-\tilde{E}_{x}^{n+1 / 2}[k]}{\Delta z}
\end{aligned}
$$

where $n$ implies time division, i.e., $t=\Delta t \cdot n$ and where the terms in parentheses represent grid point in the spatial domain, i.e., $z=\Delta z \cdot k$, respectively. In a one dimensional spatial domain, there exist two boundary points $z=0, Z$. An absorption boundary condition is necessary at both end points, $z=0, Z$ in order to keep the outgoing electrical and 
magnetic fields $E$ and $H$ from being reflected back into the target domain. This can be accomplished by setting

$$
\begin{aligned}
\tilde{D}_{x}^{n}[0] & =\tilde{D}_{x}^{n-1}[1] \\
\tilde{E}_{x}^{n}[0] & =\tilde{E}_{x}^{n-1}[1] \\
\tilde{D}_{x}^{n}[K] & =\tilde{D}_{x}^{n-1}[K-1] \\
\tilde{E}_{x}^{n}[K] & =\tilde{E}_{x}^{n-1}[K-1]
\end{aligned}
$$

where $Z=K \cdot \Delta z$. To transform Equation (3) into a time domain difference equation for implementation into FDTD, the frequency term should be replaced by the time domain representation. Noting that $1 / j \omega$ in the frequency domain is equivalent to integration in the time domain, Equation (3) becomes

$$
\tilde{D}_{x}(t)=\epsilon_{r} \tilde{E}_{x}(t)+\frac{\sigma}{\epsilon_{0}} \int_{0}^{t} \tilde{E}_{x}\left(t^{\prime}\right) d t^{\prime}+\frac{\chi_{1}}{\tau} \int_{0}^{t} \exp \left(-\frac{t^{\prime}-t}{\tau}\right) \tilde{E}_{x}\left(t^{\prime}\right) d t^{\prime} .
$$

By approximating Equation (15) as a summation in the sampled time domain, the numerical scheme can be represented by

$$
\tilde{D}_{x}^{n}=\epsilon_{r} \cdot \tilde{E}_{n}+I_{n}+S_{n}
$$

where

$$
\begin{aligned}
I^{n} & =I^{n-1}+\frac{\sigma \cdot \Delta t}{\epsilon_{0}} \cdot \tilde{E}_{x}^{n} \\
S^{n} & =\exp \left(-\frac{\Delta t}{\tau}\right) S^{n-1}+\chi_{1} \cdot \frac{\Delta t}{\tau} \cdot \tilde{E}_{x}^{n}
\end{aligned}
$$

starting with zero initial states with respect to both $\tilde{D}_{x}^{0}$ and $\tilde{E}_{x}^{0}$. Consequently, the forward problem can be implemented by the repeated scheme mentioned above:

$$
Y^{n}(\mathbf{q})=\sqrt{\frac{\mu_{0}}{\epsilon_{0}}} \tilde{E}_{x}^{n}[0]\left(J_{s}\right) .
$$

Figure 4 depicts a simulation result for a direct problem (19). A sinusoidal wave was applied to the test signal at $z_{s}=5 \cdot \Delta z$ in Equation (5):

$$
g_{s}^{n}=\sin (2 \cdot \pi \cdot f \cdot \Delta t \cdot n)
$$

where $f$ determines the frequency of the test signal. In the experiment, a sinusoidal wave of $700 \mathrm{M} \mathrm{Hz}$ is applied to a dielectric medium with parameters

$$
\epsilon_{r}=2.0, \quad \sigma=0.01, \quad \chi_{1}=2.0, \quad \tau=0.001[\mu \mathrm{sec}] .
$$

4. Stochastic Inverse Methodology. The output least square method is a conventional inverse methodology which seeks the optimal solution of

$$
J(\mathbf{q})=\min _{\mathbf{q} \in Q} \sum_{i=1}^{K}\left|Y\left(t_{i} ; \mathbf{q}\right)-Y_{d}^{i}\right|^{2} .
$$

However, it is well known that those experimental tests might not achieve good convergence results because of the complicated dependence in the related direct problem (8). In this paper, we propose a new stochastic inverse methodology for identifying those parameters. Using a Bayesian formula, the full probability model is specified by the deterministic Formula (8). The posteriori density function with respect to the set of unknown parameter vector can be represented by

$$
\pi\left(\mathbf{q} \mid \mathbf{Y}_{d}\right) \propto l\left(\mathbf{Y}_{d} \mid \mathbf{q}\right) \pi(\mathbf{q})
$$




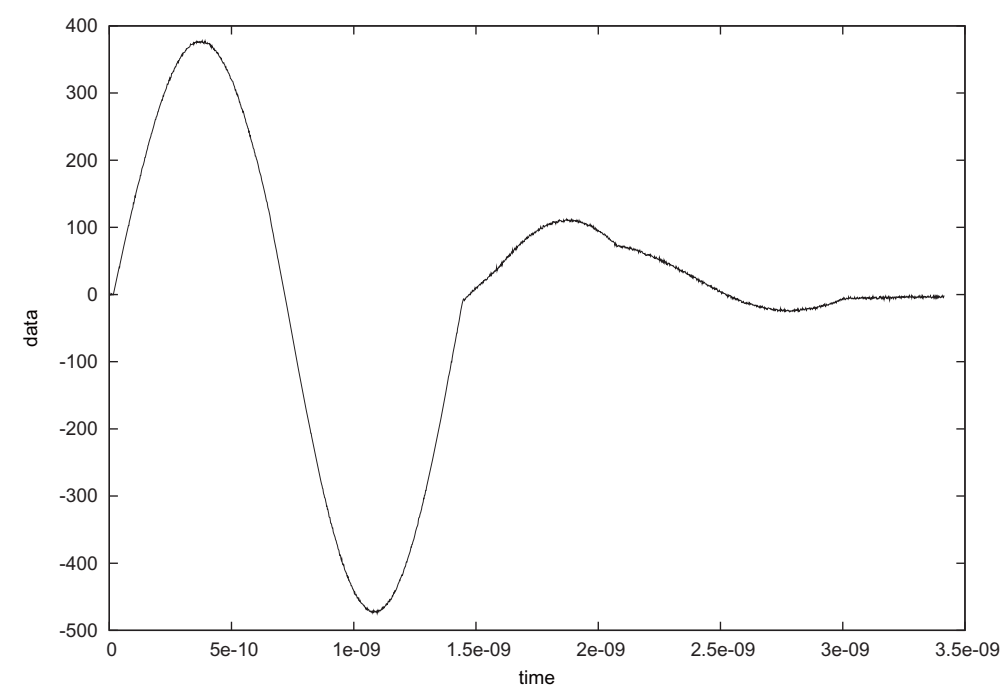

FiguRE 4. Simulation result

Suppose that measurements are made

$$
Y_{d}^{i}=Y\left(t_{i} ; \mathbf{q}\right)+\eta_{i}, \quad \eta_{i} \sim N\left(0, \xi^{2}\right), \quad(i=1,2, \ldots, K) .
$$

Then the likelihood functional can be written by the following form:

$$
l\left(\mathbf{Y}_{d} \mid \mathbf{q}\right) \propto \prod_{i=1}^{K} \frac{1}{\sqrt{2} \xi} \exp \left(-\frac{\left|Y\left(t_{i} ; \mathbf{q}\right)-Y_{d}^{i}\right|^{2}}{2 \xi^{2}}\right)
$$

In our estimation algorithm, prior distributions for unknown parameters $\pi(\mathbf{q})$ are specified as

$$
\pi(\mathbf{q})=\prod_{l=1}^{4} \pi\left(q_{l}\right)
$$

Then we specify full conditional distributions for posteriori density function through the likelihood functionals. The full conditional distribution $\pi\left(q_{l} \mid\right)(l=1,2,3,4)$ has the representation

$$
\pi\left(q_{l} \mid q_{-l}, \mathbf{Y}_{d}\right) \propto \pi\left(q_{l}\right) \prod_{i=1}^{K} \frac{1}{\sqrt{2} \xi} \exp \left(-\frac{\left|Y\left(t_{i} ; \mathbf{q}\right)-Y_{d}^{i}\right|^{2}}{2 \xi^{2}}\right)
$$

where $q_{-l}$ denotes the remaining component except $q_{l}$.

Our estimation algorithm is based on sampling procedures from the posteriori distribution from which a sample can be drawn via Markov chains. To this end, a transition kernel $p(q, \phi)$ must be constructed in such a way that the posteriori function Equation (23) is the equilibrium distribution of the chain. A simple way to implement this is to consider the reversible chains where the kernel $p$ satisfies

$$
\pi(q) p(q, \phi)=\pi(\phi) p(\phi, q) \text {. }
$$

The feature of the Metropolis-Hasting algorithm is that this reversible kernel $p$ is constructed by

$$
p(q, A)=\int_{A} k(q, \phi) \alpha(q, \phi) d \phi+I(q \in A)\left\{1-\int k(q, \phi) \alpha(q, \phi)\right\} d \phi
$$


TABLE 1. Estimation summary using MH algorithm

\begin{tabular}{|c|c|c|}
\hline \hline Quantities & True Values & Estimated Values \\
\hline$\epsilon_{r}$ & 2.00 & 1.99 \\
$\log _{10} \sigma$ & -2.00 & -1.95 \\
$\chi_{1}$ & 2.00 & 1.68 \\
$\log _{10} \tau$ & -9.00 & -9.05 \\
\hline \hline
\end{tabular}

for any subset $A$ of the parameter space. In the above expression, the acceptance probability

$$
\alpha(q, \phi)=\min \left\{1, \frac{\pi(\phi) k(\phi, q)}{\pi(q) k(q, \phi)}\right\}
$$

plays an essential role in $\mathrm{MH}$ algorithms [8]. There exist varieties of the proposal kernel functions $k$ for practical implementations. Thus the process version of the MH algorithm is given by the following steps:

Step 1: Initialize the iteration counter $j=1$ and set the initial guess of the chain $\mathbf{q}^{0}$.

Step 2: Initialize the component $i=1$.

Step 3: Move the $i$ th component of the parameter vector of the chain to a new value $\phi_{i}$ generated from the prescribed transition kernel $k_{i}\left(q_{i}^{(j-1)}, \phi_{i}\right)$.

Step 4: Calculate the acceptance probability of the move $\alpha_{i}\left(q_{i}^{(j-1)}, \phi_{i}\right)$ given by Equation (30). If the move is accepted, update the chain $q_{i}^{(j)}=\phi_{i}$. If the move is rejected, set $q_{i}^{(j)}=q_{i}^{(j-1)}$.

Step 5: Change the counter from $i$ to $i+1$ and return to Step 3 until the dimension of parameter vector, i.e., $\operatorname{dim}(\mathbf{q})=4$.

Step 6: Change the counter from $j$ to $j+1$ and return to Step 2 until convergence is reached.

5. Simulation Experiments. Throughout our numerical experiments, simulation data are generated using the direct problem in Section 3. Taking into account that actual measurement procedures can be well approximated by Equation (24), some random noise is added to the simulation data to test our inverse methodology. The second variance term in Equation (24) was provided using a conventional Gaussian random generator. The set of true parameters in the experiments is selected by Equation (21). Table 1 shows the estimated results in the experiments.

6. Conclusion. The inverse methodology arising in electromagnetic imaging was discussed within the Bayesian framework. We propose the statistical method for identifying electromagnetic material parameters on a one dimensional scattering problem of a dielectric slab. The direct problem was obtained by numerical simulation using FDTD method. The stochastic inverse problem was solved via the Metropolis-Hasting algorithm.

Acknowledgment. This material is based on research sponsored by AOARD, under agreement number No. FA2386-10-1-4076.

\section{REFERENCES}

[1] L. F. Chen, C. K. Ong, C. P. Neo, V. V. Varadan and V. K. Varadan, Microwave Electronics: Measurement and Materials Characterization, John Wiley \& Sons, West Sussex, 2004.

[2] H. T. Banks, F. Kojima and W. P. Winfree, Boundary estimation problems arising in thermal tomography, Inverse Problems, vol.6, no.4, pp.897-921, 1990. 
[3] H. T. Banks, M. W. Buksas and T. Lin, Electromagnetic Material Interrogation Using Conductive Interfaces and Acoustic Wavefronts, SIAM Publishers, Philadelphia, 2000.

[4] H. T. Banks and F. Kojima, Identification of material damage in two-dimensional domains using SQUID-based nondestructive evaluation system, Inverse Problems, vol.18, no.6, pp.1831-1856, 2002.

[5] F. Kojima, Inverse problems related to electromagnetic nondestructive evaluation, in Research Directions in Distributed Parameter Systems, R. C. Smith and M. A. Demetriou (eds.), Philadelphia, SIAM Publisher, 2003.

[6] F. Kojima and A. Ausri, Identification of stress corrosion cracking of SUS samples arising in electromagnetic nondestructive testing, J. Inv. Ill-posed Problems, vol.15, no.8, pp.799-812, 2007.

[7] W. R. Gilks, S. Richardson and D. J. Spiegelhaltger, Markov Chain Monte Carlo in Practice, Chapman \& Hall/CRC, New York, 1996.

[8] D. Gamerman, Markov Chain Monte Carlo, Stochastic Simulation for Bayesian Inference, Chapman \& Hall, New York, 1997.

[9] F. Kojima and S. Kamezaki, Identification of defect profiles using a inspection model and informative distributions, Proc. of the 35th ISCIE International Symposium on Stochastic Systems Theory and Its Applications, Ube, Yamaguchi, pp.235-240, 2004.

[10] D. M. Sullivan, Electromagnetic Simulation Using the FDTD Method, IEEE Press, New York, 2000. 